\title{
Padrão de deslocamento do camarão marinho Litopenaeus vannamei (Boone) (Crustacea, Decapoda, Penaeidae) nas fases clara e escura ao longo de 24 horas
}

\author{
Cibele S. Pontes \\ Laboratório de Estudo do Comportamento de Camarões, Departamento de Fisiologia, Universidade Federal do Rio Grande do \\ Norte. Caixa Postal 1511, 59072-970 Natal, Rio Grande do Norte, Brasil. Bolsista do CNPq. E-mail: cspontes@natal.digi.com.br
}

\begin{abstract}
Displacement pattern of marine shrimp Litopenaeus vannamei (Boone, 1931) (Crustacea, Decapoda, Penaeidae) in light and dark phases during a 24-hour period. Litopenaeus vannamei (Boone, 1931) daily behavior pattern is still poorly understood. With the aim of optimizing the management of shrimp farms through the study of this animal behavior, experiments were performed with juvenile $(7.57 \pm 1.01)$, which were marked and kept in aquariums (30 I). To record activity, the aquariums were submitted to an artificial photoperiod (light and dark phases, $12 \mathrm{~h}: 12 \mathrm{~h}$ ), with 16 shrimp $\left(33 \mathrm{~m}^{2}\right)$ monitored in each phase for 20 days, for 15-minute periods every hour. Food was provided at random times and a record kept of horizontal and vertical displacement (continuous focal), swimming and exploration behavior (instantaneous focal). The horizontal and vertical displacements of the shrimp were greater during the dark phase, with a predominance of the swimming behavior. Exploration of the substrate occurred in both phases, with a peak between five and seven hours after the lights were turned on. KEY WORDS. Applied etology; shrimp culture.
\end{abstract}

RESUMO. O padrão diário de atividade de Litopenaeus vannamei (Boone, 1931) (Crustacea, Decapoda, Penaeidae) é ainda pouco entendido. Com o objetivo de fornecer subsídios para otimizar o manejo nas fazendas de cultivo através do estudo do comportamento deste animal, foram desenvolvidos experimentos com juvenis $(7,57 \pm 1,01$ g), mantidos em aquários (30 l). Para registro da atividade, os aquários foram submetidos a fotoperíodo artificial (fase do claro e do escuro, $12 \mathrm{~h}: 12 \mathrm{~h}$ ), sendo monitorados 16 camarões $\left(33 \mathrm{~m}^{2}\right.$ ) em cada fase, durante 20 dias, em janelas de 15 min a cada hora. A alimentação foi fornecida em horários aleatórios, sendo registrados os deslocamentos horizontal e vertical (focal contínuo) e os comportamentos de natação e exploração (focal instantâneo). Os deslocamentos horizontal e vertical dos camarões foram mais acentuados na fase escura, predominando o comportamento de natação. A exploração do substrato ocorreu em ambas fases, acentuando-se entre cinco e sete horas após o acendimento das luzes.

PALAVRAS CHAVE. Carcinicultura; etologia aplicada.

A carcinicultura marinha brasileira experimentou um crescimento extraordinário a partir da introdução do camarão marinho Litopenaeus vannamei (Boone, 1931) no ano de 1982 no estado da Bahia (BuENo 1989), expandindo-se posteriormente para outros estados do país. Atualmente, utiliza-se metodologia de cultivo semi-intensivo para sua criação comercial, caracterizada por densidades populacionais moderadas (aproximadamente 30 camarões $/ \mathrm{m}^{2}$ ), oferta de alimento artificial em complemento ao natural e utilização de aeradores em horários críticos de escassez de oxigênio dissolvido.

Embora a carcinicultura seja uma indústria relativamente bem estabelecida, é importante considerar que consiste em uma atividade técnica, onde muitos dos procedimentos adotados são determinados empiricamente. VinATEA-ArAna (2004), destaca que, devido ao caráter multidisciplinar desta tecnologia, diversos tipos de conhecimentos devem convergir para a produção viável de organismos aquáticos em ambientes controlados. Neste sentido, Hungtinford (1991), afirma que há uma pressão crescente para maximização do lucro em todas as áreas onde os organismos são explorados comercialmente, e que este objetivo poderá ser atingido através de métodos desenvolvidos a partir de pesquisas na área de comportamento animal.

Fatores endógenos e exógenos têm sido investigados com relação à sua influência sobre o comportamento de camarões peneídeos. YANO et al. (1988) estudaram o comportamento reprodutivo de L. vannamei. Hill \& WASSENBERG (1992) investigaram a preferência e a quantidade de alimento ingerido pelo camarão marinho Penaeus esculentus (Haswell, 1879) de acordo com o ciclo de muda. HasheEd \& Bull (1992) avaliaram o comportamento alimentar de Melicertus latisulcatus (Kishinouye, 1896) em função da densidade populacional do cultivo e da dispersão do alimento. WASSENBERG \& Hill (1994) estudaram o 
efeito da luminosidade sobre o comportamento de emergência de oito espécies de camarões marinhos adultos de importância comercial. Nunes \& PARSONs (1998) investigaram a eficiência de manipulação e seleção do tamanho da partícula do alimento artificial pelo camarão marinho Farfantepenaeus subtilis (PérezFarfante, 1967). Primavera \& Lebata (2000) mostraram diferenças no padrão de atividade de Metapenaeus ensis (De Haan, 1844), M. latisulcatus e Fenneropenaeus merguiensis (De Man, 1888).

Existem também alguns estudos relacionados aos aspectos hierárquicos do comportamento alimentar de camarões, desencadeado a partir da oferta do alimento artificial acrescido de aminoácidos livres (geralmente betaína) como atrativos (Harpaz \& Steiner 1990, Costero \& Meyers 1993, Pittet et al. 1996, Lee \& Meyers 1996). Além disso, há pesquisas que exploram padrões de atividade em laboratório através da medição automática para a quantificação da movimentação (HINDLEY 1975, Guerao \& Ribera 1996).

De uma maneira geral, as informações específicas sobre comportamento de peneídeos, encontradas na literatura, afirmam que a maioria das espécies possui sulco adrostral e apresentam padrão de atividade similar (RoberTson et al. 1993). Tipicamente, são ativas durante a noite e se enterram durante o dia (Hindley 1975). Como exceção, espécies de camarão branco que não possuem sulco adrostral, incluindo L. vannamei, são ativas tanto durante o dia quanto à noite, e normalmente não se enterram (Pérez-Farfante 1969).

Pesquisas etológicas desenvolvidas em laboratório possibilitam a exploração da atividade associada ao exercício de ações distintas, representadas por comportamentos direcionados para objetivos específicos. Desta forma, a aplicação de conhecimentos relativos ao padrão de deslocamento associado à distribuição diária de atividades comportamentais de L. vannamei poderá vir a ser uma ferramenta útil para a melhoria do manejo nas fazendas de cultivo.

\section{MATERIAL E MÉTODOS}

Foram utilizados juvenis da espécie Litopenaeus vannamei provenientes de viveiro de cultivo, com dois meses, peso médio de 7,57 $\pm 1,01 \mathrm{~g}$, individualmente marcados com anéis de silicone no pedúnculo ocular. Neste trabalho, o emprego da nomenclatura científica para os gêneros dos camarões peneídeos segue a proposta de revisão sugerida para o grupo por PérezFARFANTE \& KensLey (1997), embora nas referências bibliográficas conste ainda a nomenclatura anteriormente utilizada.

Os experimentos foram desenvolvidos em laboratório, sendo utilizados quatro aquários $(50 \times 30 \times 40 \mathrm{~cm})$ contendo $301 \mathrm{de}$ água do mar, em sistema de recirculação de água, com aeração constante e filtração contínua através de substrato disposto no fundo do aquário, composto por lã de fibra de vidro na parte inferior e uma camada de $3 \mathrm{~cm}$ de cascalho e conchas de ostras quebradas na parte superior, que permanecia em contato direto com os camarões. Os aquários foram fechados com tampa de vidro para evitar a perda dos animais, e tiveram a face anterior dividida com fita adesiva branca de $0,5 \mathrm{~cm}$, formando oito quadrados de 12,5 cm de lado, para possibilitar a mensuração dos deslocamentos horizontal e vertical dos indivíduos.

Os aquários foram mantidos em ciclo claro/escuro de 12/12 h, utilizando-se lâmpadas fluorescente branca (15 watts) para a fase clara e incandescente vermelha $(15 \mathrm{w})$ para a escura (Rodriguez \& NAylor 1972, Hindley 1975), com intensidade luminosa de 57 lux e de 1 lux para as fases de claro e escuro, respectivamente. Em dois dos aquários, a fase clara ocorreu entre 07:00 e 19:00 h, enquanto que nos outros dois ocorreu entre 19:00 e 07:00 h, possibilitando o registro dos comportamentos em ambas as fases simultaneamente.

Os camarões foram colocados em número de quatro por aquário, eqüivalendo à densidade populacional de $33 \mathrm{~m}^{2}$, e alimentados com ração peletizada própria para seu cultivo (35\% de proteína bruta), apenas na fase em que eram observados, com o equivalente a $10 \%$ da biomassa total presente no aquário. A ração foi ofertada em comedouros de acrílico transparente $(4 \times 3 \times 2 \mathrm{~cm})$, em horários aleatórios, para evitar a interferência da oferta do alimento nas atividades dos indivíduos. As observações foram iniciadas após nove dias de adaptação dos animais às condições experimentais.

O deslocamento dos indivíduos foi registrado através de método focal contínuo, contando-se o número de quadrados percorrido pelo animal, na horizontal e na vertical, durante a observação. Foram também registradas, através de método focal instantâneo com registro a cada 60 s, as atividades: 1) Natação - deslocamento horizontal ou vertical contínuo, ou ainda suspensão na coluna d'água, através do batimento dos pleópodes e; 2) Exploração do substrato - introdução e retirada contínua dos 3 primeiros pares de pereópodes (quelados) no substrato, mantendo o cefalotórax inclinado para baixo.

Foram realizadas duas etapas experimentais, cada uma com 10 dias contínuos, onde dois aquários permaneciam no claro e dois no escuro. O acompanhamento foi realizado por dois pesquisadores simultaneamente, sendo que cada qual observava diariamente, durante 12 horas, um aquário na fase clara e outro na escura. Depois de decorrida uma hora da inversão de luminosidade, durante 15 minutos eram observados dois indivíduos de determinado aquário da fase do claro (Aquário 1 ou 2); nos 15 minutos seguintes, o processo se repetia com mais dois indivíduos do mesmo aquário. Este procedimento era repetido em seguida para um dos aquários que se encontravam na fase do escuro (Aquário 3 ou 4), havendo em seguida 60 minutos de intervalo. Desta forma, cada pesquisador observava em meia hora quatro indivíduos de determinado aquário do claro, e na meia hora seguinte quatro do escuro. Para a segunda etapa, animais em idade semelhante aos anteriores foram coletados na fazenda, realizando-se mais dez dias de observação em condições e procedimentos experimentais similares, resultando em quatro aquários e 16 indivíduos observados para cada fase, totalizando 240 horas de observação. Para efeito de análises, os horários referentes à fase do claro foram denomi- 

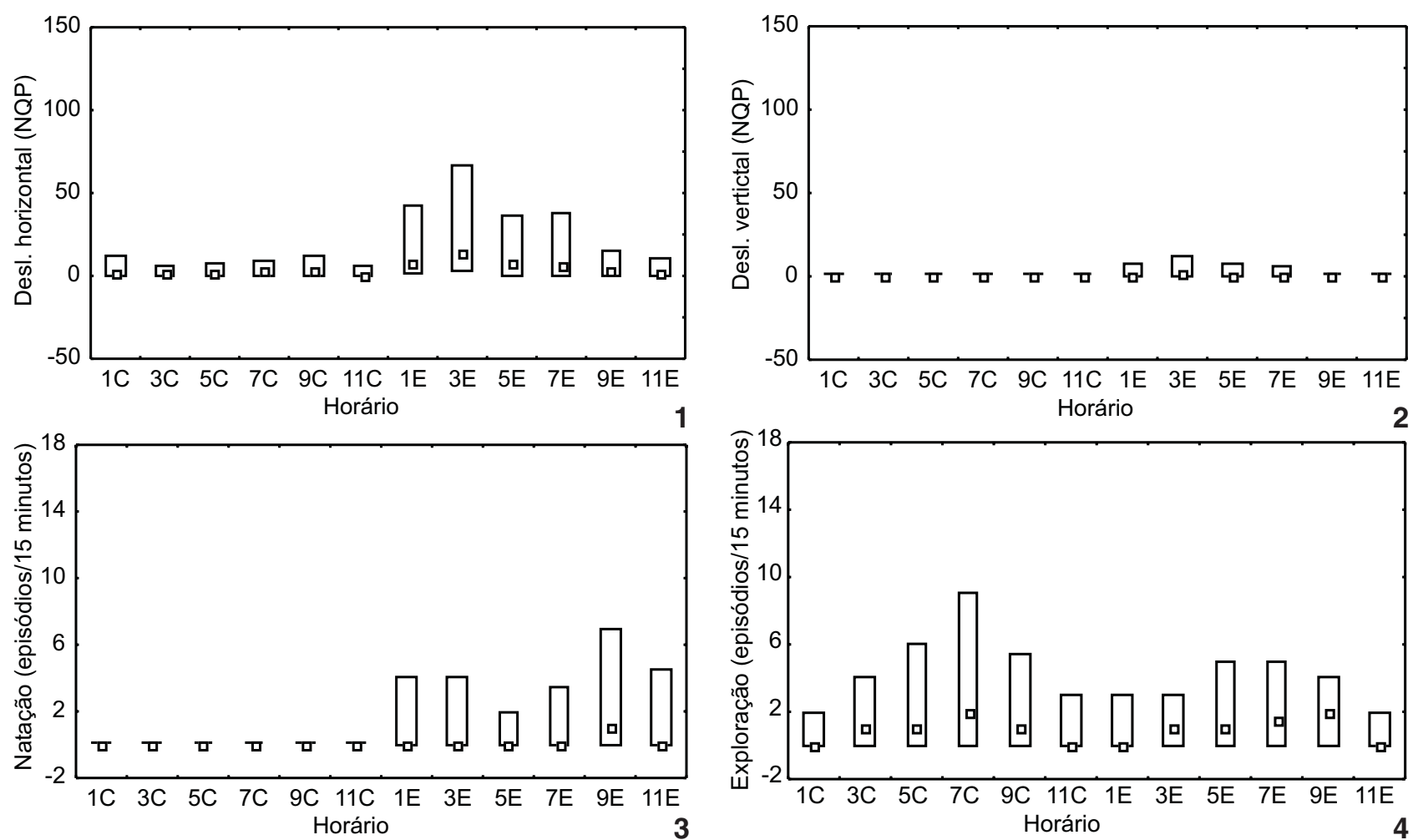

Figuras 1-4. L. vannamei de: deslocamento horizontal (1) e vertical (2) e comportamentos de natação (3) e exploração do substrato (4) de acordo com o horário de observação: (1C a 11C) fase clara, (1E a 11E) fase escura. (NQP) Número de quadrados percorridos em 15 minutos.

nados $1 \mathrm{C}$ a $11 \mathrm{C}$ e a do escuro $1 \mathrm{E}$ a $11 \mathrm{E}$, sendo os números relativos ao tempo decorrido desde a inversão da luminosidade.

As freqüências absolutas das atividades comportamentais dos camarões foram comparadas de acordo com o horário de observação (1C a 11C = horários do claro; $1 \mathrm{E}$ a $11 \mathrm{E}=$ horários do escuro). Tendo em vista a não aderência dos dados à distribuição normal (Shapiro-Wilks) e a heterogeneidade nas variâncias dos fatores (Levene), utilizou-se o teste de análise de variância não paramétrico de Kruskal-Wallis. Em caso de constatação de diferenças significativas, aplicou-se o teste post hoc U de MannWhitney para comparação dois a dois (STEEL \& TorRIE 1988). Adotou-se o nível de significância de 95\% para avaliação dos resultados, que serão graficamente representados através de sua mediana e amplitude interquartílica (75 a 25\%).

\section{RESULTADOS}

\section{Deslocamento Horizontal e Vertical}

O deslocamento horizontal dos camarões apresentou-se inferior nos horários da fase clara quando comparados aos da escura (Kruskal-Wallis, H $(11,19)=145,3 ; \mathrm{p}=0,00)$, havendo um aumento acentuado nos primeiros horários desta fase $(1 \mathrm{E}$ e 3E) e uma diminuição gradativa até o final (Fig. 1). Da mesma forma, o deslocamento vertical mostrou-se mais elevado na fase escura, sendo registrados valores mais reduzidos nos horários da fase clara (Kruskal-Wallis, H $(11,19)=246,15 ; p=0,00)$ (Fig. 2).

\section{Comportamentos de Natação e Exploração}

A natação dos animais foi mais freqüente nos horários da fase escura quando comparada à clara (Kruskal-Wallis, H $(11,19)$ =193,32; p = 0,00) (Fig. 3). Com relação à exploração do substrato, ocorreram diferenças de acordo com os horários de observação $(H(11,19)=62,82 ; p=0,00)$. Houve uma tendência ao aumento desta variável entre 5 e 7 horas após a inversão da luminosidade (nas fases clara e escura), registrando-se um pico mais significativo para a fase clara, mostrado pela elevação da amplitude de distribuição para este horário (Fig. 4).

\section{DISCUSSÃO}

Neste estudo observamos que o deslocamento, tanto horizontal quanto vertical, de L. vannamei ocorre principalmente na fase do escuro. Considerando que estas variáveis foram registradas simultaneamente aos comportamentos, podemos deduzir que a atividade locomotora deste camarão ocorre primordialmente à noite e tem como principal componente a natação. Verificamos também que a exploração do substrato 
ocorre ao longo dos horários de ambas as fases, embora seja mais freqüente na fase clara.

Com relação à atividade locomotora, Hindley (1975) investigou os efeitos do ciclo de luz sobre o camarão marinho $F$. merguiensis, expondo-o às seguintes condições de iluminação: (1) luz branca e luz vermelha alternadas (12 h/12 h), (2) luz vermelha contínua, (3) luz branca contínua. A atividade foi registrada cada vez que o animal cortava o feixe de luz de uma fotocélula. Na condição 1, a atividade foi mais elevada na fase escura, apesar de não estar restrita a ela; sob a condição 2, não foi detectada ritmicidade; sob a condição 3, apenas 1 dos 7 camarões estudados mostrou ritmicidade. Na condição 1 , a resposta de locomoção dos animais é semelhante à apresentada nesta pesquisa, embora o autor não tenha explorado qual o comportamento determinante da atividade.

Corroborando estes resultados, DALL (1986) ao estimar a rotina metabólica de juvenis de $P$. esculentus através da medição do consumo de oxigênio, afirma que estes camarões permanecem inativos durante o dia, quando há pequenas flutuações no consumo. À noite, tornam-se mais ativos, através da natação, triplicando o consumo de oxigênio. Da mesma forma, Guerao \& Ribera (1996) observaram que o padrão de atividade locomotora do camarão marinho Palaemon serratus (Pennant, 1777) mostrou ritmicidade endógena de período circadiano, com atividade máxima noturna. Trabalhos recentes relativos a ritmos em crustáceos são em sua maioria desenvolvidos com microcrustáceos. YANNICELLI et al. (2001) investigaram o ritmo de atividades dos isópodas Excirolana armata (Dana, 1852) e Excirolana braziliensis (Richardson, 1912) com relação ao nível do mar durante o ciclo da maré. MACQUARTMoulin (1999) pesquisaram a migração vertical diária e ritmo endógeno de natação em ostracodas. Tsubokura et al. (1997), analisaram os padrões sazonais de distribuição horizontal e vertical do anfípoda Trinorchestia trinitatis.

Com relação à exploração do substrato, a ausência de informações na literatura relacionadas a este comportamento, nos induz a traçar comparações indiretas com dados relativos à alimentação. Registramos que ocorre um aumento gradativo da exploração a partir do início da fase clara, que alcança valores máximos sete horas após e decai a partir daí, até alcançar os níveis mais reduzidos no último horário. Na fase escura, este comportamento ocorre em menor intensidade e de forma intercalada com a natação, distribuindo-se com relativa homogeneidade ao longo dos horários centrais. Uma vez que a exploração encontra-se diretamente relacionada à busca do alimento, indicamos como horário preferencial para a oferta da ração sete horas após o início da fase clara. Além disso, é importante considerar que uma possível oferta noturna de alimento artificial implicaria no aumento dos custos com manejo alimentar e no acréscimo de matéria orgânica ao viveiro em horário inadequado.

As pesquisas indicam que ocorre a ingestão do alimento artificial sempre que é ofertado. De acordo com McTıgue \& Feller (1989), os camarões brancos juvenis são consumidores oportu- nistas e se alimentam de qualquer material disponível no substrato. Nunes et al. (1996) verificaram que juvenis de $F$. subtilis, cultivados em sistema semi-intensivo, se alimentam tanto durante o dia quanto à noite, ocorrendo o incremento da ingestão (do alimento natural e artificial) sempre após a ração ser distribuída no viveiro. Além disso, o consumo da ração é mais intenso nos 10 minutos posteriores à oferta (NunEs et al. 1996), havendo a ocorrência mais elevada de alimento no trato digestivo 30 minutos após a sua distribuição (Nunes \& Parsons 1999). Com relação à atividade enzimática, GonzÁlez et al. (1997) observaram, para Litopenaeus shimitti (Burkenroad, 1936), picos de proteases gerais associados a todos os horários de oferta do alimento ao longo de $24 \mathrm{~h}$.

Considerando os resultados obtidos, podemos afirmar que juvenis de L. vannamei apresentam atividade tanto na fase clara quanto na escura, sendo, porém, esta atividade diferenciada em função de comportamentos específicos. A atividade locomotora ocorre na coluna d'água, através da natação, e é mais intensa à noite. Já a busca do alimento se dá através da exploração do substrato ao longo das $24 \mathrm{~h}$ do dia, com uma intensidade mais expressiva em horários característicos de maior luminosidade.

\section{AGRADECIMENTOS}

Capes, CNPq, Banco do Nordeste do Brasil, Cooperativa de Criadores Camarão do Rio grande do Norte, Fazenda de Carcinicultura Papeba e ao Departamento de Fisiologia da UFRN.

\section{REFERÊNCIAS BIBLIOGRÁFICAS}

BuENo, S.L.S. 1989. Técnicas, procedimentos e manejo para a produção de pós-larvas de camarões peneídeos: experiência vivida pela Maricultura da Bahia S.A. Brasília, Comissão Interministerial para os Recursos do Mar (CIRM Brasil), 107p.

Costero, M.T. \& S. Meyers. 1993. Evaluation of chemoreception by Penaeus vannamei under experimental condition. The Progressive Fisheries Culturist, Washington, 55 (3): 157162.

DALL, W. 1986. Estimation of routine metabolic rate in a penaeid prawn, Penaeus esculentus Haswell. Journal of Experimental Marine Biology and Ecology, Amsterdam, 96: 57-74.

González, R.; A. Suárez \& O. Carrillo. 1997. Ritmo circadiano de las proteases generales en adultos de Penaeus schmitti Efecto del horario de alimentacíon. Revista de Investigaciones Marinas, Havana, 118 (2): 162-168.

Guerao, G. \& C. Ribera. 1996. Locomotor activity patterns and feeding habits in the prawn Palaemon serratus (Pennant, 1777) (Decapoda, Palaemonidae) in the Alfa Bay, Elbro Delta, Spain. Crustaceana, Leiden, 69 (1): 101-112.

Harpaz, S. \& J. E. Steiner. 1990. Analysis of betaine induced feeding behavior on the prawn Macrobrachium rosenbergii (DeMan 1879) (Decapoda, Caridea). Crustaceana, Leiden, 58: $175-185$.

Revista Brasileira de Zoologia 23 (1): 223-227, março 2006 
Hasheed, M.A. \& C.M. Bull. 1992. Behavior on the Western king prawn Penaeus latisulcatus Kishinouye - effect of food dispersion and crowding. Australian Journal of Marine and Freshwater Research, Melbourne, 43 (4): 745-742.

Hill, B.J. \& T.J. Wassenberg. 1992. Preferences and amount of food eaten by the prawn Penaeus esculentus over the molt cycle. Australian Journal of Marine and Freshwater Research, Melbourne, 43 (4): 727-735.

Hindley, J.P.R. 1975. Effects of endogenous and some exogenous factors on the activity of juvenile banana prawn Penaeus merguiensis. Marine Biology, Berlin, 29: 1-8.

HuntingFoRd, F. 1991. The Study of Animal Behaviour. Padstow, T.J. Press, $3^{\text {rd }}$ ed., $367 \mathrm{p}$.

Lee, P.G. \& S.P. Meyers. 1996. Chemoattraction and feeding stimulation in crustaceans. Aquaculture Nutrition, Oxford, 2: $57-164$.

Macquart-Moulin, C. 1999. Diel vertical migration and endogenous swimming rhythm in Asterope mariae (Baird) and Philomedes interpuncta (Baird) (Crustacea Ostracoda Cypridinidae). Journal of Plankton Research, Oxford, 21: 20-31.

McTigue, T.A. \& R.J. Feller. 1989. Feeding of juvenile white shrimp Penaeus setiferus: periodic or continuous?. Marine Ecology Progress Series, Oldendorf/Luhe, 52: 227-233.

NunEs, A.J.P. \& G.J. PARSons. 1998. Food handling efficiency and particle size selectivity by the Southern brown shrimp Penaeus subtilis feed a dry pelleted feed. Marine and Freshwater Behavior and Physiology, Melbourne, 31 (4): 193-213.

Nunes, A.J.P. \& G.J. Parsons. 1999. Feeding levels of the Southern brown shrimp Penaeus subtilis in response to food dispersal. Journal of the World Aquaculture Society, Baton Rouge, 30 (3): 331-348.

Nunes, A.J.P.; S. Goddard \& T.C.V. Gesteira. 1996. Feeding activity patterns of the Southern brown shrimp Penaeus subtilis under semi-intensive culture in NE Brazil. Aquaculture, Amsterdam, 144: 371-386.

Pérez-Farfante, I. 1969. Western Atlantic shrimp of the genus Penaeus. Fishery Bulletin, Seattle, 67: 461-591.

Pérez-Farfante, I. \& B.F. Kensley. 1997. Penaeoid and Sergestoid
Shrimps and Prawns of the World: Keys and Diagnosis for the Families and Genera. Memories du Museum National D'Historie Naturelle, Paris, 175: 1-233.

Pittet, A.O., J.C. Ellis \& P.G. Lee. 1996. Methodology for the identification and quantitative measurement of chemical stimulants for penaeid shrimp. Aquaculture Nutrition, Oxford, 2: 75-182.

Primavera J.H. \& M.J.H.L Lebata. 2000. Size and diel differences in activity patterns of Metapenaeus ensis, Penaeus latisulcatus and Penaeus merguiensis. Marine and Freshwater Behaviour and Physiology, Victoria, 33 (3): 173-185.

RoberTson, L.; A.L. LAWRENCE \& F.L. CASTILle. 1993. Effect of feeding frequency and feeding time on Growth of Penaeus vannamei (Boone). Aquaculture and Fisheries Management, Oxford, 24: 01-06.

Rodriguez, G. \& E. NAYLor 1972. Behavioural rhythms in littoral prawns. Journal of Marine Biology Association, London, 52: 81-95.

Steel, R.G.D. \& J.H. Torrie. 1988. Bioestadística: principios e procedimientos. Mexico, McGraw-Hill, $2^{\mathrm{a}}$ ed., 622p.

Tsubokura, T.; S. Goshima \& S. Nakao. 1997. Seasonal horizontal and vertical distribution patterns of the supralittoral amphipod Trinorchestia trinitatis in relation to environmental variables. Journal of Crustacean Biology, Lawrence, 17: 13-19.

Vinatea-Arana, L. 2004. Fundamentos de Aqüicultura. Florianópolis, Universidade Federal de Santa Catarina, 349p.

WASSENBERG, T.J. \& B.J. Hill. 1994. Laboratory study of the effect of light on the emergence behavior of 8 species of commercially important adult penaeid prawns. Australian Journal of Marine and Freshwater Research, Collingwood, 45 (1): 43-50.

Yannicelli, B.; R. Palacios \& L. Gimenez. 2001. Activity rhythms of two cirolanid isopods from an exposed microtidal sandy beach in Uruguay. Marine Biology, Berlin, 138: 18-23.

Yano, I; R.A. Kanna; R.N. Oyama \& J.A. Wyban. 1988. Mating behavior in the Penaeid shrimp Penaeus vannamei. Marine Biology, Berlin, 97 (2): 171-175.

Recebido em 01.IV.2005; aceito em 23.II.2006. 